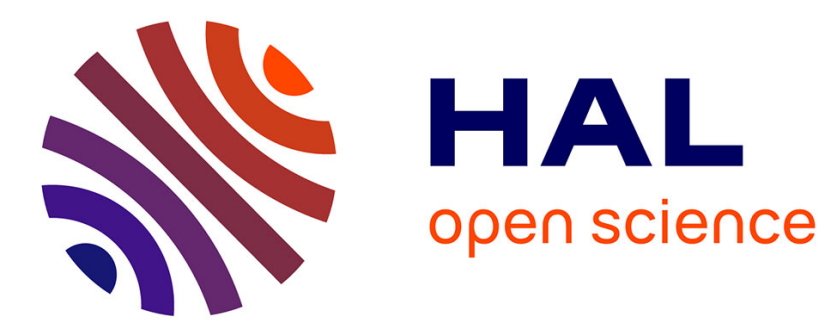

\title{
Robustness margin for piecewise affine explicit control law
}

\author{
Rajesh Koduri, Pedro Rodriguez-Ayerbe, Sorin Olaru
}

\section{To cite this version:}

Rajesh Koduri, Pedro Rodriguez-Ayerbe, Sorin Olaru. Robustness margin for piecewise affine explicit control law. 55th IEEE Conference on Decision and Control (CDC 2016), Dec 2016, Las Vegas, United States. pp.2327 - 2332, 10.1109/CDC.2016.7798610 . hal-01429228

\section{HAL Id: hal-01429228 \\ https://hal-centralesupelec.archives-ouvertes.fr/hal-01429228}

Submitted on 25 Mar 2017

HAL is a multi-disciplinary open access archive for the deposit and dissemination of scientific research documents, whether they are published or not. The documents may come from teaching and research institutions in France or abroad, or from public or private research centers.
L'archive ouverte pluridisciplinaire HAL, est destinée au dépôt et à la diffusion de documents scientifiques de niveau recherche, publiés ou non, émanant des établissements d'enseignement et de recherche français ou étrangers, des laboratoires publics ou privés. 


\title{
Robustness margin for piecewise affine explicit control law
}

\author{
Rajesh Koduri ${ }^{1}$ Pedro Rodriguez-Ayerbe ${ }^{1}$ and Sorin Olaru ${ }^{1}$
}

\begin{abstract}
Classical robustness margin i.e., gain margin and phase margin, considers the gain variation and phase variation of the model preserving the stability of the closed loop. In this paper, an attempt to find the same kind of margin for a piecewise affine (PWA) controller is done. This controller usually obtained in explicit model predictive control (MPC) is defined over a convex region of the state space $\mathcal{X}$. Starting from the invariance property of the closed loop obtained with a discrete dynamic model and PWA controller in a convex region of the state space, we calculate two robustness margin preserving this invariance property. The first one will be denoted as gain margin corresponding to the variation of the gain of the model guaranteeing the invariance. The second one, that we call, the robustness margin against first order neglected dynamics correspond to the smallest first order neglected dynamic allowed in the system preserving the invariance property.
\end{abstract}

\section{INTRODUCTION}

Explicit model predictive control (MPC) belongs to a class of constrained model based optimization methods whose solution is synthesized by a set of piecewise affine (PWA) dynamics. The idea of the explicit MPC [1][3][2] is to express the on-line quadratic problem in the control design into a multi-parametric Quadratic Programming(mpQP) problem and to reduce the computation of the optimal control inputs to a simple evaluation of a control law stored in a lookup table. Such control laws can be easily implemented for real-time system with fast dynamics and relative small statespace models. Though explicit control law has favorable advantages over the standard MPC, it also comes up with high computational cost for higher order systems and/or for large prediction horizon.

From the analysis point of view, it is important to take into account the capacity of the control law that can cope with disturbances, neglected dynamics or uncertain parameters. This characteristic is termed in control theory as robustness of the controller. In the context of robustness analysis of explicit controller, very few contributions have been made. Few noticeable works include an analysis procedure proposed in [4] and [5] handles the robustness/fragility of the positive invariance for the dynamics affected by uncertain parameters. On the other hand, there is a substantial work on the robustification of the explicit controllers. The reference [6]

\footnotetext{
*The work leading to these results has received funding from the People Programme (Marie Curie Actions) of the European Unions Seventh Framework Programme (FP7/2007-2013) under REA grant agreement no 607957 (TEMPO)

${ }^{1}$ The Authors are with L2S, CentraleSupelec, CNRS, LIPS, Paris Saclay University, 91192, Gif-Sur-Yvette, France. rajesh.koduri@centralesupelec.fr, pedro.rodriguez-ayerbedsupelec.fr, sorin.olaru@centralesupelec.fr
}

shows how to improve the robustness of the controller taking disturbances into account in the design phase. In a different perspective [7] presented an robust explicit predictive control synthesis which accounts for uncertainties based on dynamic programming.

In this paper, we consider the inherent robustness properties of explicit predictive control described as piecewise affine (PWA) control laws for a class of linear discrete time systems. First, we present a numerical method to compute a gain margin set for a discrete-time system stabilized by a continuous PWA dynamics with respect to the invariance property. The desired gain margin set is a polytope which characterize for variations of system gains preserving the invariant characteristics of the controller. Second, we analyze for the dynamic system affected by first order neglected dynamics. The robustness margin of the controller is defined by a set which characterize for a range of parameters preserving the invariance of the controller.

The paper is organized as follows: In section III, the system description for explicit model predictive control is briefly discussed. Then in section IV, we introduce some important notations and tools which are used throughout the paper. In section $\mathrm{V}$, we discuss about the gain margin set for a given PWA control law with two examples. In section IV, we present the robustness margin of the PWA control law affected by the first order neglected dynamics. Finally, in section VII, we sum up the paper with conclusion.

\section{BASIC NotATIONS}

This section addresses some basic notations and definitions. A vector is noted $x \in \mathbb{R}^{n}, x=\left[\begin{array}{lll}x_{1} & \cdots & x_{n}\end{array}\right]^{T}$ and a matrix $A \in \mathbb{R}^{n \times m}, A=\left[a_{i j}\right]$. An identity matrix is represented by $I_{n}$, where the subscript $n$ denotes the dimension of that matrix. The sets $\mathbb{R}, \mathbb{R}_{+}, \mathbb{Z}, \mathbb{N}$ and $\mathbb{N}_{>0}$ denote set of real numbers, set of non-negative real numbers, set of integers, set of non-negative integers, set of positive integers, respectively. We denote $\mathbb{R}^{n}$ a Euclidean space and $x \in \mathbb{R}^{n}$ a vector with $n$ elements.

A polyhedron is the (convex) intersection of a finite number of open or closed half-spaces and a polytope is a bounded and closed polyhedron. A mapping function $f: \mathbb{R}^{n} \rightarrow \mathbb{R}^{m}$ is said to be positively homogeneous of the first degree, if $f(\alpha x)=\alpha f(x), \forall \alpha \in \mathbb{R}_{+}$and $\forall x \in \mathbb{R}^{n} . \mathbf{1}$ is a vector with its elements equal to 1 . For a $N \in \mathbb{N}_{+}, \mathcal{I}_{N}$ denotes the set of integers, $\mathcal{I}_{N}:=\left\{i \in \mathbb{N}_{+} \mid i \leq N\right\} . \operatorname{Proj}_{R^{c 2}} S$ is a projection mapping of a set $S$ onto a subset, $S \subset \mathbb{R}^{c 1}$ $\rightarrow \mathbb{R}^{c 2}$ for $c 1>c 2$ and will be considered to operate on the first $c 2$ coordinates of $\mathbb{R}^{c 1}$. Finally, convh denotes the convex hull. 


\section{SYSTEM DESCRIPTION}

Consider a linear discrete-time system given by,

$$
\begin{aligned}
x(k+1) & =A x(k)+B u(k) \\
y(k) & =C x(k)
\end{aligned}
$$

Where, $x(k) \in \mathbb{R}^{n}, u(k) \in \mathbb{R}^{m}$ and $y(k) \in \mathbb{R}^{s}$ denote the state vector, input variables and output variables respectively at time $k$. The constraints on the system states and input variables are represented by,

$$
\begin{array}{r}
X=\left\{x: H_{x} x \leq h_{x}, \quad H_{x} \in \mathbb{R}^{n_{x} \times n}, h_{x} \in \mathbb{R}^{n_{x}}\right\} \\
U=\left\{u: H_{u} u \leq h_{u}, \quad H_{u} \in \mathbb{R}^{m_{u} \times m}, h_{x} \in \mathbb{R}^{m_{u}}\right\}
\end{array}
$$

The state and input constraints are bounded polytopic sets. For explicit MPC, the quadratic cost function of the standard MPC with the terminal and stage costs is transformed into a multi-parametric Quadratic Programming (mp-QP) problem [2]. Considering a discrete-time system given by (1) subject to constraints (2), the equivalent mp-QP problem is given as

$$
\begin{array}{r}
J(x, z)=\min _{z} \frac{1}{2} z^{T} H z+x^{T} F^{T} z+\frac{1}{2} x^{T} Y x \\
\text { s.t } \quad G z \leq W+S x .
\end{array}
$$

The optimal solution for (3) is represented by a finite set of affine functions defined over the polyhedral partition of the set $\mathcal{X}$, where $\mathcal{P}_{N}(\mathcal{X})=\left[\mathcal{X}_{1}, \mathcal{X}_{2} \cdots \mathcal{X}_{N}\right]$ is called the polyhedral partition of the set $\mathcal{X}$. The polyhedral $\mathcal{X}_{i} \subset \mathbb{R}^{n}$ are called critical regions or components of the partition. The polyhedral regions $\mathcal{X}_{i}$ are non overlapping i.e., $\mathcal{X}_{i} \cap \mathcal{X}_{j}=\emptyset$, for $i \neq j$. The non-overlapping property ensures the unique solution for the state $x(k) \in \mathcal{X}_{i}, \forall i \in \mathcal{I}_{N}$. Two neighboring regions $\mathcal{X}_{i}$ and $\mathcal{X}_{j}$ share some vertices or facets and thus the optimal solution over the set $\mathcal{X}$ exhibits the continuity property.

Definition 1: A mapping function

$$
f_{\text {pwa }}:\left\{\mathbb{R}^{n} \rightarrow \mathbb{R}^{m} \mid A_{i} x+b_{i}, \forall x \in \mathcal{X}_{i}, i \in \mathcal{I}_{N}\right\}
$$

defined over the polyhedral partition of the set $\mathcal{X}$ is called a piecewise affine function of the polyhedral partition.

The explicit control law, solution of (3), is synthesized in terms of the piecewise affine function defined over the polyhedral partition of the set $\mathcal{X}$ and it can be described by

$$
u_{p w a}(x(k))=F_{i} x(k)+g_{i}, \quad \forall x(k) \in \mathcal{X}_{i} .
$$

Definition 2: A closed and bounded set $\mathcal{P} \subset \mathcal{X}$ is called positively invariant with respect to the system (1) in closed loop with the control law $u_{\text {pwa }}(x(k))$ if for any $x(0) \in \mathcal{P}$, it follows $x(k) \in \mathcal{P}, \forall k \in \mathbb{Z}_{[1 ; \infty]}$.

\section{PRELIMINARIES}

This section introduces few matrices which will be used throughout the paper. The vertex representation of the polyhedral sets $\mathcal{X}$ and $\mathcal{X}_{i}$ are given by

$$
\begin{array}{r}
\mathcal{X}=\operatorname{convh}\left\{w_{1}, w_{2} \cdots w_{r}\right\} \\
\mathcal{X}_{i}=\operatorname{convh}\left\{w_{i 1}, w_{i 2} \cdots w_{i r_{i}}\right\}
\end{array}
$$

A matrix $W \in \mathbb{R}^{n \times r}$ stores the vertices of the polyhedron $\mathcal{X} \in \mathbb{R}^{n}$.

$$
W=\left[w_{1}, w_{2}, \cdots, w_{r}\right]
$$

We introduce a matrix $V \in \mathbb{R}^{n \times \sum_{i=1}^{N} r_{i}}$ to store the vertices of all the partition in the state space $\mathcal{X}_{i} \subset \mathcal{X}, \forall i \in \mathcal{I}_{N}$.

$$
V=\left[w_{11}, w_{12}, \cdots, w_{i r_{i}}, \cdots, w_{N r_{N}}\right]
$$

Like stated before, neighboring partitions have vertices in common, and therefore, the repeated vertices are removed from the matrix $V$ and therefore the matrix $V$ contains nonidentical column vectors.

$$
V=\left[w_{1}, w_{2}, \cdots, w_{p}\right] \in \mathbb{R}^{n \times p}
$$

With the help of affine mapping $f(x(k))=u_{p w a}(x(k))$, a matrix $U \in \mathbb{R}^{m \times p}$ is used to store the control input for each column of matrix $V$.

$$
U=u_{p w a}(V)
$$

\section{GAIN MARGIN}

In the following, we describe the gain margin set for the system (1) stabilized with the help of a state feedback explicit control laws. The construction of the gain margin set is based on vertex representation of the regions forming the partition $\mathcal{X}$.

Definition 3: Consider a discrete time linear system (1) with a continuous PWA control law (5), such that the state space set $\mathcal{X}$ is positively invariant. The Gain Margin is represented by the set $\mathcal{K} \subset \mathbb{R}^{m}$, such that $x(k+1)=$ $A x(k)+B\left(I_{m}+\operatorname{diag}\left(\delta_{K}\right)\right) u_{p w a}(x(k)) \in \mathcal{X}, \forall x(k) \in \mathcal{X}$ and $\delta_{K} \in \mathcal{K}, \delta_{K} \in \mathbb{R}^{m}$.

The set $\mathcal{K} \subset \mathbb{R}^{m}$ is a set which contains the input channels gain variations $\delta_{K}$ such that for any point inside the set $\mathcal{K}$, the invariance characteristics of the set $\mathcal{X}$ are preserved.

Theorem 1: Consider a discrete-time linear system (1) with a piecewise affine state feedback control law defined in (5). The gain margin $\mathcal{K}$ of the controller is defined by the set,

$$
\mathcal{K}=\cap_{q=1}^{p} \mathcal{K}_{q}
$$

Where $\mathcal{K}$ represents the gain margin set and $\mathcal{K}_{q}$ the local gain margin for the vertex $w_{q}$ for some $q \in \mathcal{I}_{p}$.

Proof: Starting from the PWA closed loop dynamics assuring the invariance of the set $\mathcal{X}$ given by,

$$
x(k+1)=A x(k)+B u_{p w a}(x(k)) \in \mathcal{X}
$$

with the addition of $\delta_{K}$ in (12), $\forall \delta_{K} \in \mathcal{K}$ we obtain:

$$
x(k+1)=A x(k)+B\left(I_{m}+\operatorname{diag}\left(\delta_{K}\right)\right) u_{p w a}(x(k)) \in \mathcal{X}
$$

It is also possible to exploit the structure of the PWA closedloop dynamics, by introducing the parametric variations on the control gains preserving the invariance and boundedness properties of the controller. Suppose that $\hat{u}=u_{\text {pwa }}(x(k))+$ $\delta_{u}$, where the term $\delta_{u} \in \mathbb{R}^{m}$ describes admissible control input variations,

$$
x(k+1)=A x(k)+B u_{p w a}(x(k))+B \delta_{u} \in \mathcal{X}
$$


By relating (13) and (14) we obtain:

$$
\begin{array}{r}
A x(k)+B u_{p w a}(x(k))+B \delta_{u}= \\
A x(k)+B\left(I_{m}+\operatorname{diag}\left(\delta_{K}\right)\right) u_{p w a}(x(k))
\end{array}
$$

And,

$$
u_{p w a}(x(k))+\delta_{u}=\left(I_{m}+\operatorname{diag}\left(\delta_{K}\right)\right) u_{p w a}(x(k))
$$

By rewriting (16) with analogy to each column vectors of the matrix $V, \forall q \in \mathcal{I}_{p}$ (vertices in the set $\mathcal{X}_{i}$ ), we obtain:

$$
\delta_{u_{q}}=\operatorname{diag}\left(\delta_{K_{q}}\right) u_{p w a}\left(w_{q}\right)
$$

The admissible input $\delta_{u_{q}}$ belongs to a set $\Delta \mathcal{U}_{q} \subset \mathbb{R}^{m}$ and $\delta_{K_{q}} \in \mathcal{K}_{q}$. A matrix $M_{q}=\operatorname{diag}\left(u_{p w a}\left(w_{q}\right)\right) \in \mathbb{R}^{m \times m}$ is uniquely defined based on the value of the control action $u_{p w a}\left(w_{q}\right)$ such that

$$
\Delta \mathcal{U}_{q} \supseteq M_{q} \mathcal{K}_{q}
$$

Exploiting the polyhedral structure of the admissible input variations,

$$
\Delta \mathcal{U}_{q}=\left\{u: \mid \hat{H}_{u} u \leq \hat{h}_{u}\right\}
$$

where $\hat{H}_{u} \in \mathbb{R}^{d_{u} \times m}$ and $\hat{h}_{u} \in \mathbb{R}^{d_{u}}$ one can obtain the local set of gain variation for the vertex $w_{q}$ as:

$$
\mathcal{K}_{q}=\left\{z \in \mathbb{R}^{m} \mid \exists u \in \Delta \mathcal{U}, M_{q} z=u\right\}
$$

Finally,

$$
\mathcal{K}_{q}=\left\{z \in \mathbb{R}^{m} \mid \hat{H}_{u} M_{q} z \leq \hat{h}_{u}\right\}
$$

The collection of sets $\mathcal{K}_{q}$ are independent of each vertices of the set in $\mathcal{X}_{i}$ and the intersection of these independent set gives the global set $\mathcal{K}$. In order to compute $\mathcal{K}_{q}$, we first need to compute explicitly the sets $\Delta \mathcal{U}_{q}$.

Remark 1: Even if no control gain variation is admissible in (13), the null vector $\mathbf{0}_{m} \in \mathcal{K}$ is admissible as long as it corresponds to the set invariance of the original PWA control law.

\section{A. Construction of $\Delta \mathcal{U}_{q}$ set:}

In this subsection, a description for the $\Delta \mathcal{U}_{q}$ set is constructed based on forward mapping of the vertex in the PWA partition.

Theorem 2: Consider a linear discrete-time system (1) stabilized by a piecewise affine control law (5). The set $\Delta \mathcal{U}_{q}$ of admissible input variations at the vertex $w_{q}$ is obtained by

$$
\Delta \mathcal{U}_{q}=\operatorname{Proj}_{U} \mathcal{H}_{q}
$$

$U \in \mathbb{R}^{m}$ denotes the input constraint set. The polyhedral set $\mathcal{H}_{q}$ is described by:

$$
\mathcal{H}_{q}=\left\{\begin{array}{c}
\left(\delta_{u}, \lambda\right) \in \mathbb{R}^{m} \times \mathbb{R}^{r}, \text { and }[A B]\left[\begin{array}{c}
x(k) \\
u_{p w a}(x(k))
\end{array}\right] \\
+B \delta_{u}=W \lambda
\end{array}\right\}
$$

Proof: Let us recall the equation (14) which resumes the positive invariance for the set $\mathcal{X}$,

$$
\left[\begin{array}{ll}
A & B
\end{array}\right]\left[\begin{array}{c}
x(k) \\
u_{p w a}(x(k))
\end{array}\right]+B \delta_{u} \in \mathcal{X}
$$

From the definition of invariance, (24) can be expressed using the vertices of the set $\mathcal{X}$ as convex combination and corresponding variables $\lambda=\left[\lambda_{1}, \lambda_{2}, \cdots, \lambda_{r}\right]$ and equality constraints $\mathbf{1}^{T} \lambda=1$ such that

$$
\left[\begin{array}{ll}
A & B
\end{array}\right]\left[\begin{array}{c}
x(k) \\
u_{p w a}(x(k))
\end{array}\right]+B \delta_{u}=W \lambda .
$$

\section{B. Computation of $\Delta \mathcal{U}_{q}$ set:}

Based on the value of the control action $u_{p w a}\left(w_{q}\right)$. Consider the objective of finding the $\Delta \mathcal{U}_{q}$ set for each column vector of the matrix $V$ independently, $\forall q \in \mathcal{I}_{p}$.

$$
A x(k)+B u_{p w a}(x(k))+B \delta_{u}=W \lambda
$$

Where $\lambda=\left[\lambda_{1}, \lambda_{2}, \cdots, \lambda_{r}\right]$ and $\mathbf{1}^{T} \lambda=1$ with $\lambda_{i} \geq 0$ $\forall i \in \mathcal{I}_{r}$. Clearly (26) can be represented by a system of linear equalities with the variable vector $\Gamma$, where the vector $\Gamma$ contains the required variable $\delta_{u}$ along with the variables $\left[\lambda_{1} \cdots \lambda_{r}-1\right]$.

$$
\begin{array}{r}
\Gamma=\left[\delta_{u}, \lambda_{1}, \lambda_{2}, \cdots, \lambda_{r-1}\right] \in \mathbb{R}^{m+r-1} \\
\lambda_{r}=1-\sum_{i=1}^{r-1} \lambda_{i}, \quad \lambda_{i} \geq 0 .
\end{array}
$$

A linear programming problem with (26) and (27) as linear equality constraints can be setup,

$$
\left[\left[\begin{array}{c}
B(1,:) \\
\vdots \\
B(n,:)
\end{array}\right]-\hat{W}\right] \Gamma=w_{r}-\left(A w_{q}+B u_{p w a}\left(w_{q}\right)\right)
$$

where $\hat{W}$ is defined by the notion $W=\left[w_{1} \cdots w_{r}\right]$

$$
\hat{W}=\left[w_{1}-w_{r}, \cdots, w_{r-1}-w_{r}\right] \in \mathbb{R}^{n \times r-1}
$$

The system of linear equalities (28) is defined in the form $H \Gamma=h$, where $H \in \mathbb{R}^{n \times(m+r-1)}$ and $h \in \mathbb{R}^{n \times 1}$, and has the solution

$$
\Gamma=H_{z} t+h_{z},
$$

where $H_{z}=\operatorname{null}(H)$ is nothing but the orthonormal basis for the null space of matrix $H$ and $h_{z}$ is the solution set for the linear programming problem. Subsequently, we establish two polyhedral sets from the matrices $H_{z}, h_{z}$ with a variable $t$. The first polyhedral set corresponds to the required set $\Delta \mathcal{U}_{q}$. Recalling the non-negativity constraints defined in (27), the second polyhedral is defined with the non-negativity property of the elements in the solution vector $\Gamma(m+1: m+r-1)=\left[\lambda_{1} \cdots \lambda_{r-1}\right]^{T} \geq 0$,

$$
\begin{array}{r}
\mathcal{H}^{(1)}=\left\{t \mid H_{z}^{(1)} t \leq h_{z}^{(1)}\right\} \\
\mathcal{H}^{(2)}=\left\{t \mid-H_{z}^{(2)} t \leq h_{z}^{(2)}\right\} .
\end{array}
$$

The matrices appearing in (30) are related to a decomposition $H_{z}^{(1)}=H_{z}(1: m,:), h_{z}^{(1)}=h_{z}(1: m), H_{z}^{(2)}=$ $H_{z}(m+1: m+r-1,:)$ and $h_{z}^{(2)}=h_{z}(m+1: m+r-1)$. And finally the set $\Delta \mathcal{U}_{q}$ is nothing but a linear transformation of the set $\mathcal{H}^{(2)}$,

$$
\Delta \mathcal{U}_{q}=H_{z}^{(1)} \mathcal{H}^{(2)}+h_{z}^{(1)}
$$


The vertices of $\Delta \mathcal{U}_{q}$ is computed by applying the transformation to all the vertices of the set $\mathcal{H}^{(2)}$.

Corollary 1: The set $\mathcal{K}_{q}$ representing the gain matrix set is a polyhedral $\forall q \in \mathcal{I}_{p}$.

Proof: The set $\mathcal{H}_{q}$ and $\Delta \mathcal{U}_{q}$ used in the description for $\mathcal{K}_{q}$ are polyhedral and by this virtue $\mathcal{K}_{q}$ inherits this property.

Remark 2: we note the analogy between the results reported in [5] and (22). The work of [5] focuses on the computation of fragility margin which is characterized for the parametric variations in the space of $F_{i}$ for each individual regions. However, in the present framework we interpret those findings to compute the variations in the control input for each individual vertices in the set $\mathcal{X}$.

Remark 3: Regarding the computation of the set $\mathcal{K}$, one need to solve $p$ LP problems with a set of linear equations given in (28), where $p$ is the number of non-identical vertices in the set $\mathcal{X}$.

\section{Examples:}

Single Input system:

Consider a linear discrete time system,

$x(k+1)=\left[\begin{array}{cc}1.4 & 0 \\ 0.8 & -1.1\end{array}\right] x(k)+\left[\begin{array}{l}0.5 \\ 0.7\end{array}\right] u(k)$,

$y(k)=\left[\begin{array}{ll}1 & 0\end{array}\right] x(k)$. The weight matrices $Q=\left[\begin{array}{ll}1 & 0 \\ 0 & 1\end{array}\right]$ and $R=1$. The prediction horizon chosen is 2 . The input constraint $-5 \leq u(k) \leq 5$ and the output constraint $-5 \leq y(k) \leq 5$. Solving the mp-QP yields 13 controllers. The gain margin set $\mathcal{K}$ in (11) is computed and the value of $\delta_{K}$ lies between $[-0.2178,0.3051]$.

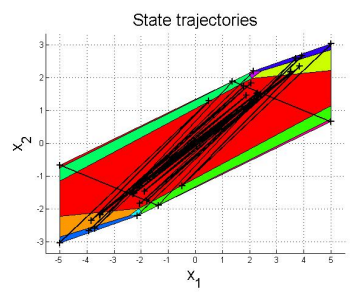

Fig. 1: state trajectories for $\delta_{K}=0.3$

State trajectories are simulated in closed loop with $\delta_{K}$ value such that $x(k+1)=A x(k)+B\left(1+\delta_{K}\right) u_{\text {pwa }}(x(k))$.

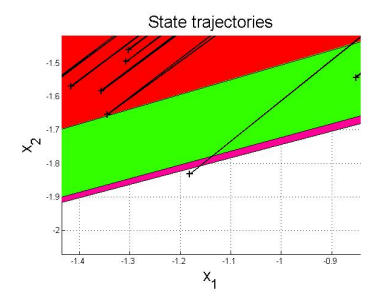

Fig. 2: state trajectories for $\delta_{K}=-0.25$

Figure 1 shows the state trajectories for $\delta_{K}=0.3$ which is a value inside the computed gain margin and it is observed that the trajectories are inside the invariant set thus confirming the theoretical result. From figure 2, it is noticeable that the controller is no more invariant for $\delta_{K}=-0.25$.

Multi Input system: Consider a linear discrete system with two inputs and two outputs,

$x(k+1)=\left[\begin{array}{ccc}1.2 & -1 . & 0 \\ 0 & -1.2 & 0.5 \\ 0.2 & 0.4 & 0\end{array}\right] x(k)+\left[\begin{array}{cc}1.0 & 0.2 \\ 0.5 & 0 \\ 0 & 0.7\end{array}\right] u(k)$,

$y(k)=\left[\begin{array}{ccc}1 & 0 & 0 \\ 0 & 0 & 1\end{array}\right] x(k)$. The weight applied on the control inputs and state vectors are $Q=\left[\begin{array}{lll}5 & 0 & 0 \\ 0 & 1 & 0 \\ 0 & 0 & 1\end{array}\right]$ and $R=\left[\begin{array}{cc}0.5 & 0 \\ 0 & 1\end{array}\right]$. The input constraint $\left[\begin{array}{l}-2 \\ -2\end{array}\right] \leq u(k) \leq\left[\begin{array}{l}2 \\ 2\end{array}\right]$ and the output constraint $\left[\begin{array}{l}-2 \\ -2\end{array}\right] \leq y(k) \leq\left[\begin{array}{l}2 \\ 2\end{array}\right]$. The prediction horizon chosen is 2 . The resulting controller has 37 regions. Figure $3 a$ shows the set $\mathcal{K}$ for (5) assuring the invariance of the set $\mathcal{X}$. However, the invariance property of the controller is fragile to unmeasured input perturbation. For instance, choosing $\delta_{K}=\operatorname{diag}(-0.01,-0.01)$ results in the controller loosing the invariance. To prove the positive side of gain margin analysis, a new explicit controller is chosen by adapting the gain with an admissible variation from the set $\mathcal{K}$, shown in figure $3 \mathrm{a}$, and which preserves its definition domain over the set $\mathcal{X}$.

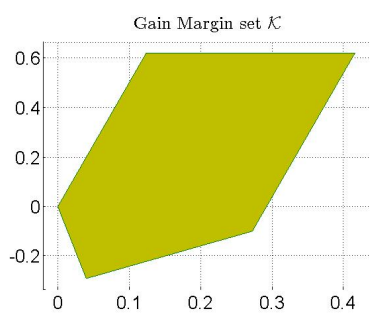

(a) $\mathcal{K}$ set for $u_{p w a}(x(k))$

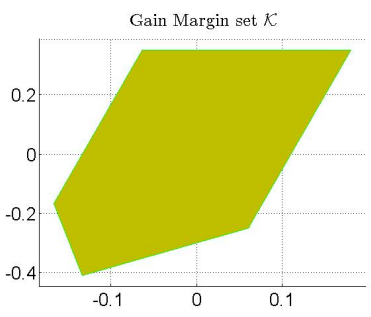

(b) $\mathcal{K}$ set for $u_{p w a}^{\mu}(x(k))$
Fig. 3: Gain Margin set

The new controller is adapted in a straightforward manner to $u_{p w a}^{\mu}(x(k))=\left(I_{m}+\operatorname{diag}\left(\delta_{K}\right)\right) *\left(F_{i} x(k)+g_{i}\right) \forall i \in \mathcal{I}_{N}$ and $\delta_{K}=\operatorname{diag}(0.2,0.2)$. Subsequently, we computed the gain margin set for this controller and present an illustration of its corresponding admissible variation in figure $3 \mathrm{~b}$. It is noticeable from the figure that the new controller is more robust to input disturbances and practically we obtained a translation of the point corresponding to the nominal controller without altering the shape of the set $\mathcal{K}$. The new controller also violates the input constraints but the violation can be omitted by saturating the control inputs.

\section{FIRST ORDER NEGLECTED DYNAMICS}

In the following, we analyze the robustness of the controller (5) defined over the polyhedral set $\mathcal{X}$ affected by a first order neglected dynamics in closed loop. Our approach 
is to present an admissible set for the dynamic variations of the neglected parameters or variables assuring the invariance of the set $\mathcal{X}$.

\section{A. Problem Formulation}

In this section, a problem is formulated for the first order dynamics that perturb the closed loop system as shown in figure 4 .

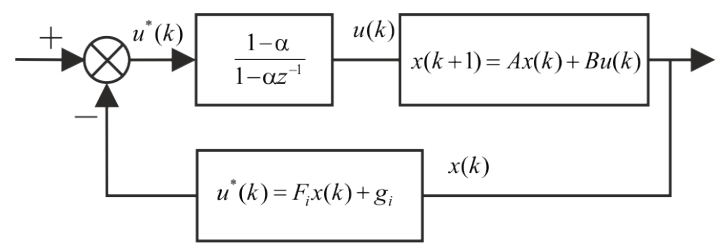

Fig. 4: Closed loop system with first order neglected dynamics

The neglected dynamics can be represented by a first order equation and it can be written in the form,

$$
\begin{aligned}
x_{I}(k+1) & =\alpha x_{I}(k)+(1-\alpha) u^{*}(k) \\
u(k) & =\alpha x_{I}(k)+(1-\alpha) u^{*}(k)
\end{aligned}
$$

The neglected dynamics in closed loop model can be described by,

$$
x(k+1)=A x(k)+\alpha B x_{I}(k)+B(1-\alpha) u^{*}(k)
$$

The augmented model is

$$
\begin{array}{r}
x_{e}(k+1)=A_{e} x_{e}(k)+B_{e} u(k) \\
y_{e}(k)=C_{e} x_{e}(k)
\end{array}
$$

and,

$$
\begin{gathered}
x_{e}=\left[\begin{array}{c}
x \\
x_{I}
\end{array}\right], A_{e}=\left[\begin{array}{cc}
A & \alpha B \\
0_{1 \times n} & \alpha
\end{array}\right] \\
B_{e}=\left[\begin{array}{c}
B(1-\alpha) \\
1-\alpha
\end{array}\right], C_{e}=[C, 0]
\end{gathered}
$$

where $x_{e} \in \mathbb{R}^{n_{e}}, A_{e} \in \mathbb{R}^{n_{e} \times n_{e}}$ and $B_{e} \in \mathbb{R}^{n_{e} \times m}$.

Starting from a piecewise affine control (5) defined over the polyhedral partition of the set $\mathcal{X}$ with $\mathcal{X}_{i} \in \mathcal{X} \forall i \in \mathcal{I}_{N}$ for the linear discrete time system (1) the aim is to investigate the robustness of the control synthesis (5) for the system (34) and (35) affected by the neglected dynamics. The objective being to find the largest set of $\alpha$ assuring the invariance of the set $\mathcal{X}$, first we construct vertices for the augmented state with the help of the vertices in the set $\mathcal{X}_{i} \forall i \in \mathcal{I}_{N}$. Second, we analyze the robustness of the control synthesis (5) with respect to the invariance characteristics for the set $\mathcal{X}$.

\section{B. Construction of vertices for the augmented state}

Before entering into the technical details of the construction, we discuss about the extension of vertices for the augmented state.

Recall the vertices of the set $\mathcal{X}$ and $\mathcal{X}_{i}$ defined by the column vectors of matrices (7) and (9). The piecewise affine control law $u_{\text {pwa }}(x(k))$ for the column vectors of the matrix $V$ is given by the matrix $U$ (10). The construction of vertices for the augmented state $x_{I}$ can be done in two ways.

- Method 1: Initialize the values of the state $x_{I}(k)$ for each vertex corresponding to the column vectors of matrix $V$ with the piecewise affine control law. For instance, $w_{I_{p}}=u_{p w a}\left(w_{p}\right) \forall p \in \mathcal{I}_{p}$.

- Method 2: Initialization of the state $x_{I}(k)$ for each vertex corresponding to the column vectors of matrix $V$ can be carried out with the help of minimum and maximum values of the control input.

Method 2 produces twice the number of vertices as compared to method 1. For each column vector of matrix $V$, we obtain two different vertices i.e., the values of augmented state vector $x_{e}$ for each non-identical vertices in $\mathcal{X}_{i}$ is $\left[w_{p}, u_{\text {min }}\right]$ and $\left[w_{p}, u_{\max }\right] \forall p \in \mathcal{I}_{p}$. In the following, we investigate the robustness problem for method 1 .

\section{Controller for extended state system}

The controller for the extended model (34) can be represented by,

$$
u_{p w a}^{e}\left(x_{e}(k)\right)=F_{i}^{e} x_{e}(k)+g_{i}^{e}
$$

where $F_{i}^{e}=\left[F_{i} 0\right] \in \mathcal{R}^{m \times n_{e}}$ and $g_{i}^{e}=g_{i} \in \mathcal{R}^{m \times 1}$.

The controller has no impact on the state $x_{I}$. This is just an extension of (5) to accommodate the extended state and therefore the characteristics of the original controller (5) are preserved.

D. An admissible set for the first order neglected dynamics:

We introduce few matrices which will be used in the construction. The extended model (34)-(35) can be written as a convex combination of the extreme realization obtained with $\alpha=0$ and $\alpha=1$.

For $\alpha=0$,

$$
A_{1}^{e}=\left[\begin{array}{cc}
A & 0_{n \times m} \\
0_{m \times n} & 0_{m \times m}
\end{array}\right], \quad B_{1}^{e}=\left[\begin{array}{c}
B \\
1_{m \times m}
\end{array}\right]
$$

For $\alpha=1$,

$$
A_{2}^{e}=\left[\begin{array}{cc}
A & B \\
0_{m \times n} & 1_{m \times m}
\end{array}\right] \quad B_{2}^{e}=\left[\begin{array}{c}
0_{n \times m} \\
0_{m \times m}
\end{array}\right]
$$

Therefore,

$$
A_{e}=(1-\alpha) A_{1}^{e}+\alpha A_{2}^{e}, B_{e}=(1-\alpha) B_{1}^{e}+\alpha B_{2}^{e}
$$

Since our objective follows the computation of maximum $\alpha$ value assuring the invariance of the set $\mathcal{X}$, we just need the state and input values in order to produce for the state vector $x(k+1)$ in (33).

$$
\begin{aligned}
& A_{1}=\left[\begin{array}{ll}
A & 0_{n \times m}
\end{array}\right] \in \mathbb{R}^{n \times n_{e}} \quad A_{2}=\left[\begin{array}{ll}
A & B
\end{array}\right] \in \mathbb{R}^{n \times n_{e}} \\
& B_{1}=B \text { and } B_{2}=\left[0_{n \times m}\right] .
\end{aligned}
$$

Similar to (9), we introduce a matrix $V_{e}^{(1)} \in \mathbb{R}^{n_{e} \times p}$ which stores the vertices of the state vector $x(k)$ and the PWA control input associated with it for all the non-identical vertices in $\mathcal{X}_{i} \in \mathbb{R}^{n}, i \in \mathcal{I}_{N}$ in order to create a matrix that contains the vertices for the state vector $x_{e}$.

$$
V_{e}^{(1)}=[V, U]
$$


In a second step, the values of the state vector $x_{e}(k+1)$ are found by exploiting (40) and (36) for column vectors of the matrix $V_{e}^{(1)}$ in a closed loop formulation. This leads to the matrix $V_{e}^{(2)} \in \mathbb{R}^{n_{e} \times p}$.

$$
V_{e}^{(2)}=A V_{e}^{(1)}+B U
$$

Similar to the notation in (10), a PWA image for the matrix $V_{e}^{(2)}$ can be found with the control law (36) and stored in a matrix $U_{e}^{(2)} \in \mathbb{R}^{m \times p}$.

$$
U_{e}^{(2)}=u_{p w a}^{e}\left[V_{e}^{(2)}\right]
$$

Definition 4: Consider a linear discrete time system affected by the first order neglected dynamics (34)-(35) and stabilized via a PWA state feedback control law (36). A margin for first order neglected dynamics is characterized by a set $\Omega^{\alpha} \in \mathbb{R}^{m}$ which contains the values of parameter $\alpha$ (for each input channel) such that the invariance property of the set $\mathcal{X}$ is assured.

Theorem 3: Consider the extended system (34) subject to first order neglected dynamics stabilized by a piecewise affine control law (36). The admissible set of parameters for the neglected dynamics is given as the projection,

$$
\Omega^{\alpha}=\operatorname{Proj}_{\alpha} \mathcal{T}
$$

where $\mathcal{T}$ denotes the polyhedral set:

$$
\mathcal{T}=\left\{\begin{array}{c}
(\alpha, \Gamma) \in \mathbb{R} \times \mathbb{R}^{r \times p} \mid(1-\alpha)\left(A_{1} V_{e}^{(2)}+\right. \\
\left.B_{1} U_{e}^{(2)}\right)+\alpha\left(A_{2} V_{e}^{(2)}+B_{2} U_{e}^{(2)}\right)=W \Gamma
\end{array}\right\}
$$

Proof: If $\Omega^{\alpha}$ describes admissible set for neglected dynamics $\forall \alpha \in \Omega^{\alpha}$ then $\left((1-\alpha) A_{1}+\alpha A_{2}\right) x_{e}(k)+((1-$ $\left.\alpha) B_{1}+\alpha B_{2}\right) u_{p w a}^{e} \in \mathcal{X}, \forall k \in \mathbb{Z}_{[1 ; K]}$.

We remark that the computation of the admissible set $\Omega^{\alpha}$ corresponds to the variations between the nominal model (1) and the model affected by the neglected dynamics (34).

$$
\begin{array}{r}
(1-\alpha)\left(A_{1} x_{e}(k+1)+B_{1}\left(F_{i}^{e} x_{e}(k+1)+g_{i}^{e}\right)\right)+ \\
\alpha\left(A_{2} x_{e}(k+1)+B_{2}\left(F_{i}^{e} x_{e}(k+1)+g_{i}^{e}\right)\right) \in \mathcal{X}
\end{array}
$$

By replacing $x^{e}(k)$ with the column elements of the matrix $V_{e}^{(2)}$ and similarly the PWA function $u_{p w a}^{e}=F_{i}^{e} x^{e}(k+1)+$ $g_{i}^{e}$ with the column vectors of matrix $U_{e}^{(2)}$. Equation (45) is further modified into:

$$
\begin{array}{r}
(1-\alpha)\left(A_{1} V_{e}^{(2)}(:, q)+B_{1} U_{e}^{(2)}(:, q)\right)+ \\
\alpha\left(A_{2} V_{e}^{(2)}(:, q)+B_{2} U_{e}^{(2)}(:, q)\right) \in \mathcal{X}
\end{array}
$$

For $q \in \mathcal{I}_{p}$, by representing the state vector $x \in \mathcal{X}$ as the convex combination of column vector of the matrix $W$, one can write $\sum_{l=1}^{r} \gamma_{l} W(:, l)$ and $\sum_{l=l}^{r} \gamma_{l}=1$ and introducing this term in (46) obtain

$$
\begin{gathered}
(1-\alpha)\left(A_{1} V_{e}^{(2)}(:, q)+B_{1} U_{e}^{(2)}(:, q)\right)+ \\
\alpha\left(A_{2} V_{e}^{(2)}(:, q)+B_{2} U_{e}^{(2)}(:, q)\right)=W \gamma
\end{gathered}
$$

with a vector $\gamma=\left[\gamma_{1}, \cdots, \gamma_{r}\right] \in \mathbb{R}^{r}$.

If (47) holds for one column vector of matrix $V_{e}^{(2)}$, then it holds for all the column vectors of the matrix $V_{e}^{(2)}$ and with the image of the piecewise affine defined by the matrix $U_{e}^{(2)}$.

Equation (47) can be written as,

$(1-\alpha)\left(A_{1} V_{e}^{(2)}+B_{1} U_{e}^{(2)}\right)+\alpha\left(A_{2} V_{e}^{(2)}+B_{2} U_{e}^{(2)}\right)=W \Gamma$

with $\Gamma \in \mathbb{R}^{r \times p}$ and the proof is completed.

\section{Example}

For the single input system given in the previous section, the margin for the first order neglected dynamics is represented by the set $\Omega^{\alpha}=[\{0,0.267\}]$. The invariance property of the controller is preserved for any input filter with parameters in these values.

\section{CONCLUSION}

In this work the gain margin set assuring the invariance of the closed loop obtain for a linear discrete time system controlled by a piecewise affine control law has been computed. For a discrete time system affected by first order neglected dynamics, a robustness margin has been also deduced assuring the invariance property. It is worth to be mentioned that this analysis procedure proposed does not extend to analysis to the convergence (asymptotic stability properties). Future work will include the robustness analysis for PWA control law assuring the contractivity.

\section{REFERENCES}

[1] A. Bemporard, M. Morrari, V. Dua and E. N. Pistikopoulos, The explicit linear quadratic regulator for constrained systems, Automatica, vol. 38(1), pp. 3-20, 2002.

[2] A. Alessio and A. Bemporad, A survey on explicit model predictive control, In Nonlinear model predictive control: Springer 2009, pp. 345-369.

[3] A. Grancharova, T. A. Johansen, Explicit Nonlinear Model Predictive Control Theory and Applications, Springer Science \& Business Media, vol. 429, 2012.

[4] S. Olaru, N. A. Nguyen, G. Bitsoris, P. Rodriguez-Ayerbe and M. Hovd, Explicit robustness margins for discrete-time linear systems with PWA control, System Theory, Control and Computing (ICSTCC), 2013 17th International Conference, pp. 380-385, Oct. 2013.

[5] N. A. Nguyen, S. Olaru, G. Bitsoris and P. Rodriguez-Ayerbe, Explicit fragility margins for PWA control laws of discrete-time linear systems, Control Conference (ECC), European, Strasbourg, 2014, pp. 14501455, 2014.

[6] P. Rodrguez-Ayerbe and S. Olaru, On the disturbance model in the robustification of explicit predictive control, International Journal of Systems Science, vol 44(5), 2013

[7] P. N. Efstratios, N. P. Faísca, K. I. Kouramas, P. Christos, Explicit robust model predictive control, In Proceedings of the international symposium on advanced control of chemical processes, ADCHEM 09, 2009.

[8] C. Dorea and J. C. Hennet, $(\mathrm{a}, \mathrm{b})$-invariant polyhedral sets for linear discrete-time systems, Journal of Optimization Theory and Applications, vol. 103(3), pp. 521-542, 2005.

[9] M. Hovd, S. Olaru and G. Bitsoris, Low Complexity constraint control using contractive sets, In World congress, vol.19, pp. 2933-2938, 2014

[10] M. Herceg, M. Kvasnica, C. N. Jones and M. Morari, Multi-Parametric Toolbox 3.0, Proc. of the European Control Conference, pp. 502-510, July 2013.

[11] A. Suardi, S. Longo, E. C. Kerrigan and G. A. Constantinides, Robust explicit MPC design under finite precision arithmetic, 19th IFAC World Congress, vol. 47(3), pp. 2939-2944, 2014.

[12] M. Kvasnica, J. Lófberg and M. Fikar, Stabilizing polynomial approximation of explicit MPC, Automatica, vol. 47(10), pp. 2292-2297, 2011. 